\title{
Parameter Estimation of a Skill Evaluation Model
}

\author{
Kazuo Kawada \\ Department of Technology and Information Education, Hiroshima University, \\ 1-1-1 Kagamiyama, Higashi-Hiroshima, Hiroshima 739-8524, Japan \\ Teruyuki Tamai \\ Matsuyama City Kamogawa Junior High School, \\ 2-7-19 Kamogawa, Matsuyama, Ehime 791-8004,Japan \\ Toru Yamamoto \\ Division of Electrical, System and Mathematical Engineering, Hiroshima University, \\ 1-4-1 Kagamiyama, Higashi-Hiroshima, Hiroshima 739-8527,Japan \\ E-mail:kawada@hiroshima-u.ac.jp,E-mail:tama.wr@gmail.com,yama@hiroshima-u.ac.jp \\ www.hiroshima-u.ac.jp
}

\begin{abstract}
In this study, the aim is to construct a "teacher-student model" for optimal skill acquisition. To support the optimal skill acquisition, modeling and estimation of an individual learning process are very important. The first-order system with time delay is introduced as an individual learning process model based the control engineering approach. These system parameters included in the skill evaluation model are estimated by using a real-coded genetic algorithm. In order to evaluate the effectiveness of the proposed scheme, it is employed for the parts classification task.
\end{abstract}

Keywords: Skill evaluation model, Teacher-student model, Learning process, First-order system, Real-coded genetic algorithm, Parameter estimation.

\section{Introduction}

In order to effectively promote skill acquisition in the learning and the work, it is necessary to grasp the learning situation of individual learners and workers and to appropriate support based on the learning situation. From the initial learning performance, if the future learning process can be predicted based on individual learning characteristics, the optimum learning support according to individuals can be performed. In response to that problem, the modeling was proposed in which skill evaluation was regarded as "first order + time delay" system in the control engineering ${ }^{1}$. The meaning of each parameters of the time constant $T$, time delay $L$ and system gain $K$ in the model of skill evaluation model was considered. However, it has not been studied how to estimate parameters of skill evaluation model from initial learning data. In this paper, the estimate method of the skill evaluation model is proposed by using real-coded genetic algorithm ${ }^{2}$ (GA) from measured trial data. In addition, the results of estimating the parameters of the skill evaluation model from the initial learning performance data are also discussed. 


\section{Parameter Estimation of Skill Evaluation Model}

\subsection{Fitness value considering characteristics of skill evaluation model}

In order to estimate parameters of the skill evaluation model, it is necessary to eliminate variations in task execution time. Such variations can be divided into two factors: task factors and learner's factors. Variations due to task factors are changed due to the order of tasks to be started from among many tasks. On the other hand, the variation due to the learner's factors changes due to accidental delay in execution time or trial and error. Therefore, the estimation of the skill evaluation model excluding learner's factor variations is considered using "part classification task" with small variations due to the task factors.

\subsection{TKL model}

As a requirement of the skill evaluation model, the following is necessary.

1) It is exponential.

2) The parameters can be determined individually. Generally, the controlled object given in the higher order system is described as the "first-order + time delay" system, and the control system is designed by rough system characteristics. In this paper, it is difficult to make the skill evaluation model strict. However, if the reference is set at the learning start, it can be represented by the model shown in Fig. 1.

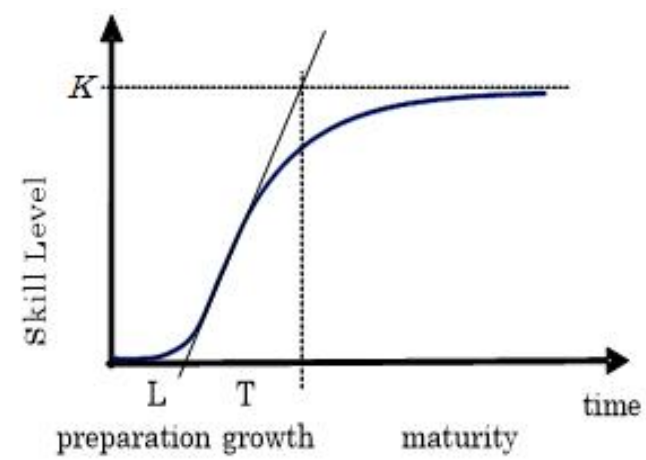

Fig. 1 Skill levels and learning time.

"First order + time delay" system (TKL model) is described in the following equation.

$$
G(s)=\frac{K}{1+T s} e^{-L s}
$$

where $T$ denotes the time constant, $K$ denotes the system gain, and $L$ denotes the time delay. The method to calculate these parameters $(T, K$ and $L)$ from measured trial data.

The procedure for obtaining $T, K$ and $L$ from the measured trial data is as follows.

[1] Measure each response time $T_{R}[1 \ldots n]$ from 1 time to $n$ times.

[2] $a, b$ and $c$ included in the following Eq.(2) are calculated by the real-coded GA. The estimated response time required for the $n$-th task is as follows:

$$
\hat{T}_{R}[n]=\hat{a}+\hat{b} e^{-\hat{c} t[n]}
$$

where $t[n]$ represents the real learning time from 1 time to $n$ times, and is expressed by the following equation.

$$
t[n]=\sum_{i=1}^{n}\left(T_{R}[i]-\hat{a}\right)
$$

[3] Calculate $T, K$ and $L$ by the following equation, using $a, b$ and $c$ obtained using the real-coded GA.

$$
\begin{aligned}
T & =\frac{1}{\hat{c}}, \\
L & =T_{R}[1]-\hat{a} \\
K & =\hat{b} e^{-\hat{c} L}
\end{aligned}
$$

\section{Parameter Estimation using Genetic Algorithm}

The parameters $a, b$ and $c$ of skill evaluation model are arranged as cells included in a string, whose structure is shown in Fig. 2. These parameters included in the string are given by real values. The real-coded GA is employed, which is explained as follows.

(i) Initialization

The generation number $G$ is set, and the initial individuals are produced with random real-codes within the initial domain which is set in advance. Here, the number of population is set as $N$.

(ii) Selection

The fitness value $f(l)$ is calculated which is given by

$$
f(l)=1 /\left\{1+\sum_{k=1}^{t_{\text {end }}}\left(\hat{T}_{R}[k]-T_{R}[k]\right)^{2} .\right.
$$

where $\hat{T}_{R}$ and $T_{R}$ respectively denote the learning time and the estimated learning time by the parameters of skill evaluation model $a, b$ and $c$. Each individual $P_{l}$ is arranged in order, 
based on the fitness value. Then, $\alpha$ percent individuals with superior fitness values are selected, and saved in the next generation.

(iii) Crossover

The $(100-\alpha)$ percent remaining are generated by the crossover. Two individuals, $P_{a}$ and $P_{b}$ are chosen from among the superior $\alpha$ percent, and new individuals $P_{c}$ and $P_{d}$ are generated by employing the following procedure:

$$
\begin{aligned}
& P_{c}(i)=P_{\text {sup }}(i)-\frac{\left|P_{a}(i)-P_{b}(i)\right|}{4}, \\
& P_{d}(i)=P_{\text {sup }}(i)+\frac{\left|P_{a}(i)-P_{b}(i)\right|}{4}
\end{aligned}
$$

where $P_{\text {sup }}$ in Eq.(8) and Eq.(9) refers to the individual with the superior fitness value, i.e., $P_{a}$ or $P_{b}$. Note that this procedure is employed for every cell included in $P_{a}$ and $P_{b}$.

(iv) Mutation

Of all individuals which are randomly selected and given by the crossover, $\beta$ percent are chosen and replaced with randomly determined values within the initial domain.

(v) Update

The procedure from (i) through (iv) is repeated for generations.

This procedure is summarized in Fig. 3.

\section{Experimental Results for Part Classification Task}

The parameters $T, K$ and $L$ of the skill evaluation model of the parts classification are calculated and considered. The fourteen kinds of parts shown in Fig. 4 were placed in the mixed state, and tasks for classifying the same parts were prepared. The number of each part is shown in Table 1. The subject was a university student, and he had no experience of executing parts classification task. Subject took 3 minutes break and between each task, and carries out the total of 11 tasks and measured the time required for each task. The execution results are shown in Table 2. It was reduced from the required time 179 [s] in the first task to 54 [s] in the 11 th task. The skill level is the time obtained by subtracting the minimum required time $a$ from the response time in each task in Table 2, and the learning time to each task is the skill level added.

$a, b$ and $c$ were searched by using the real-coded GA, $a=0.13, b=238$ and $c=0.0017$ were obtained. $T, K$ and $L$ were obtained from Eq.(4)-Eq.(6), and
$T=588, K=176$ and $L=178$ were obtained by using these values $a, b$ and $c$. Fig. 5 is shown from these $T, K$ and $L$.

Next, Fig.6 shows the relationship between the initial learning point and the estimated parameters. From this figure, it is clear that $T, K$ and $L$ are almost possible to estimate at the initial learning 4 points.

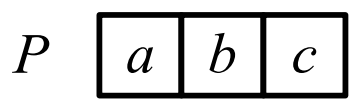

Fig. 2 Structure of the GA string.

Initial Individual Population

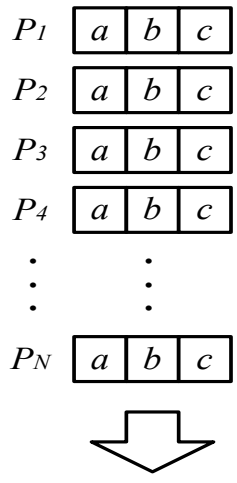

Selection

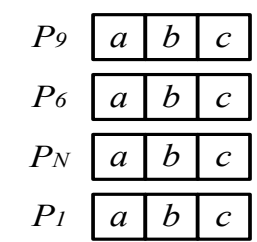

Fitness function $f(1)$

8.7 High

6.6

$-\div--:----\div-1$

$P_{3}$\begin{tabular}{|l|l|l|}
\hline$a$ & $b$ & $c$ \\
\hline
\end{tabular}

0.3 Low

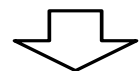

Crossover

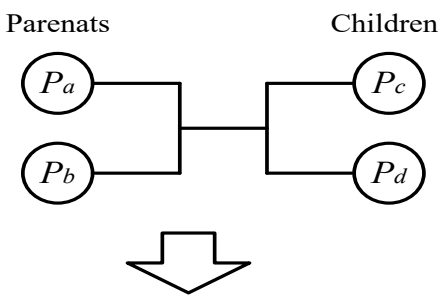

Mutation

$\beta \%$ cells are replaced with random values. \begin{tabular}{l|l|l|l|}
$P^{\text {Child }}$ & $a$ & $b$ & $c$ \\
\hline
\end{tabular}

Fig. 3 Calculation flow of the evolutionary computation. 


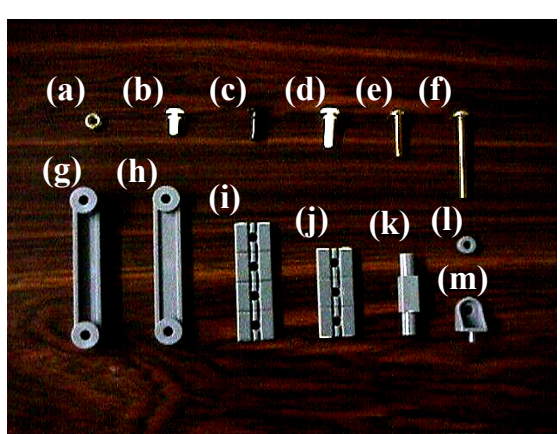

Fig. 4 Parts to be classified.

Table 1 Number of parts.

\begin{tabular}{c|c}
\hline Fastener Components & Number \\
\hline \hline (a) & 20 \\
\hline (b) & 20 \\
\hline (c) & 20 \\
\hline (d) & 20 \\
\hline (e) & 10 \\
\hline (f) & 10 \\
\hline (g) & 10 \\
\hline (h) & 20 \\
\hline (i) & 10 \\
\hline (j) & 5 \\
\hline$(\mathrm{k})$ & 20 \\
\hline (l) & 20 \\
\hline (m) & 20 \\
\hline
\end{tabular}

Table 2 Number of task and response time.

\begin{tabular}{c|c|c}
\hline Number of Task & $t[\mathrm{~s}]$ & $T_{R}[\mathrm{~s}]$ \\
\hline \hline 1 & 179 & 179 \\
\hline 2 & 310 & 131 \\
\hline 3 & 441 & 131 \\
\hline 4 & 534 & 93 \\
\hline 5 & 612 & 78 \\
\hline 6 & 697 & 85 \\
\hline 7 & 754 & 57 \\
\hline 8 & 813 & 59 \\
\hline 9 & 867 & 54 \\
\hline 10 & 917 & 50 \\
\hline 11 & 971 & 54
\end{tabular}

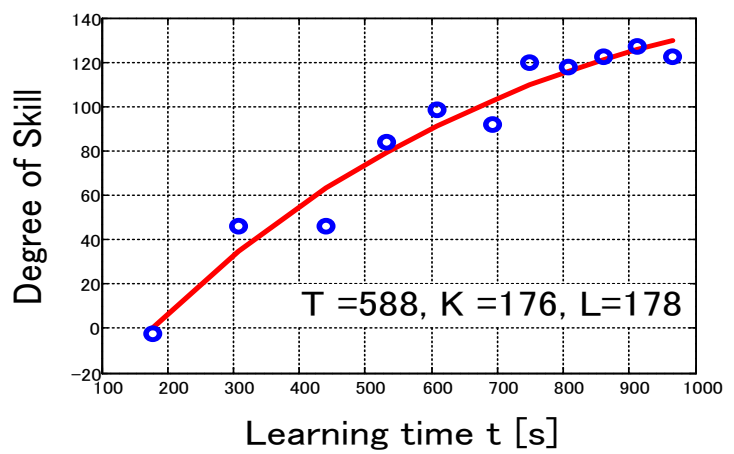

Fig. 5 Estimated skill evaluation model.
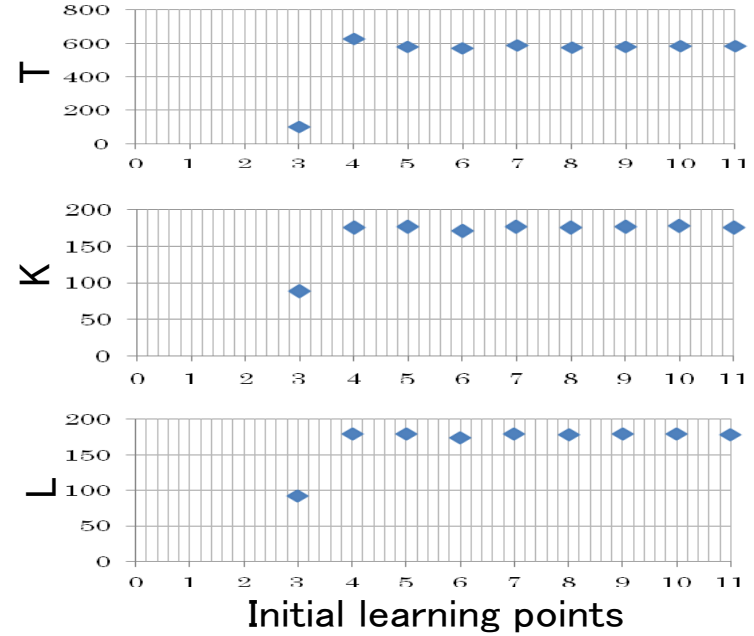

Fig. 6 Relationship between initial learning points and estimated parameters.

\section{Conclusion}

In order to realize an optimum learning support system by individual skill evaluation in the learning process, the validity of the skill evaluation model based on the control engineering approach was discussed. Next, by applying to the classification work of parts, parameters of the skill evaluation model are estimated from the results of the initial learning, and the effectiveness was shown. In the future, it will be applied to the computational learning etc. and plan to develop the learning support system.

This work was supported by JSPS KAKENHI Grant Numbers JP16K12760, JP16H02921.

\section{References}

1. M. Nagamatsu, T. Usuzaka, K. Kawada, T. Yamamoto and, Y. Yamane: Consideration on a Model between Teacher and Student Based on Control Engineering Approach, IEEJ Transactions on Electronics, Information and Systems, Vol.134, No.10, (2014) pp.1537-1542. (in Japanese)

2. R. Loren and D. B. Benson (eds.), Introduction to String Field Theory, 2nd edn. (Springer-Verlag, New York, 1999).

3. M. F. Mason, M.I. Norton, J.D. Van Horn, D.M. Wegner, S.T. Grafton, and C.N. Macrae: Wandering Minds: The Default Network and Stimulus-Independent Thought, Science, 315, (2007) pp.393-395.

4. W. Hasenkamp, C.D. Wilson-Mendenhall, E. Duncan, L.W. Barsalou, Mind wandering and attention during focused meditation: A fine-grained temporal analysis of fluctuating cognitive states, Neuroimage, 59, (2012) pp.750-760. 